\title{
O cuidado em saúde mental centrado na pessoa: uma experiência na atenção primária à saúde
}

\author{
Care at the mental health centered in persons: an experience in primary health care
}

Atención de salud mental centrada en las personas: una experiencia em atención primaria de salud

Leidiely Gomes Moraes ${ }^{1 *}$, Larissa de Almeida Rézio ${ }^{1}$, Samira Reschetti Marcon.

\section{RESUMO}

Objetivo: Relatar a experiência de uma discente do curso de graduação em enfermagem quanto ao desenvolvimento de intervenções em saúde mental direcionadas a uma adolescente no âmbito da Atenção Primária à Saúde. Relato de Experiência: Trata-se de um relato de experiência desenvolvido a partir de vivências em uma Estratégia de Saúde da Família de uma capital brasileira. Os cuidados direcionaram-se a uma adolescente acompanhada pela enfermagem. Durante cinco encontros, entre atendimentos na unidade, visita domiciliária e discussão interprofissional, foram realizadas intervenções centradas nas suas demandas, utilizando o referencial da Atenção Centrada na Pessoa e modelo de atenção psicossocial. A partir disso, o cuidado foi construído de modo dialogado, resultando em melhorias na adolescente referentes à afetividade, humor e, maior empenho em interagir com a acadêmica. Considerações Finais: Analisar esse processo, como discente, permitiu uma reflexão acerca da possibilidade de atuação do enfermeiro referente aos cuidados com a saúde mental na Atenção Primária à Saúde, à luz do modelo de atenção psicossocial e Atenção Centrada na Pessoa.

Palavras-chave: Assistência centrada no paciente, Saúde mental, Atenção primária à saúde, Assistência integral à saúde.

\section{ABSTRACT}

Objective: To report the experience of a graduate student in nursing regarding the development of mental health interventions for an adolescent in the field of Primary Health Care. Study Experience: This is an experience report developed from experiences in a Family Health Strategy of a Brazilian capital. The care was directed to an adolescent accompanied by nursing. During five meetings, between unit care, home visit, and interprofessional discussion, interventions centered on their demands were carried out, using the referential of Person-Centered Care and the psychosocial care model. From this, care was built in a dialogic way, resulting in improvements in the adolescent regarding affectivity, humor and, greater commitment to interact with the academic. Final Considerations: Analyzing this process, as a student, allowing a reflection about the possibility of the nurse's action regarding mental health care in Primary Health Care, in light of the model of psychosocial care and Person-centered Care.

Keywords: Patient-centered care, Mental health, Primary health care, Comprehensive health care.

\section{RESUMEN}

Objetivo: Reportar la experiencia de un estudiante de postgrado en enfermería acerca del desarrollo de intervenciones de salud mental para un adolescente en el campo de la Atención Primaria de Salud. Experiencia de Estudio: Este es un informe de experiencia desarrollado a partir de las experiencias en una Estrategia de Salud Familiar de una capital brasileña. El cuidado fue dirigido a un adolescente acompañado por enfermería. Durante cinco reuniones, entre la atención en la unidad, visita a domicilio y debate interprofesional, se han llevado a cabo intervenciones centradas en sus demandas, utilizándose del modelo de referencia de la atención centrada en la persona y de la atención psicosocial. La atención se ha construido de manera dialógica, resultando en la mejora del adolescente en la afectividad, humor y, mayor compromiso al interactuar con el académico. Consideraciones Finales: El análisis de dicho proceso, como estudiante, ha permitido reflexionar acerca de la posibilidad de la actuación de la enfermera en materia de salud mental en la Atención Primaria de Salud, a la luz del modelo de atención psicosocial y de la Atención Centrada en la Persona.

Palabras clave: Atención dirigida al paciente, Salud mental, Atención primaria de salud, Atención integral de salud.

1 Universidade Federal de Mato Grosso (UFMT), Cuiabá - MT. *E-mail: leidielymoraes@gmail.com 


\section{INTRODUÇÃO}

A Estratégia de Saúde da Família (ESF) visa o rearranjo da Atenção Primária à Saúde (APS) por meio de uma assistência ainda mais assertiva, devendo ofertar atendimento a usuários, família e grupos de pessoas (BRASIL, 2017). Quando se presta tais serviços, é preciso considerar, inclusive, a execução de planos de cuidado que abrangem a realidade de cada indivíduo. Nesse sentido, a Atenção Centrada na Pessoa (ACP) diz respeito a uma diretriz da APS que norteia intervenções de acordo com a demanda do usuário, estimulando a autonomia e saúde, levando em consideração fatores sociais e familiares (BRASIL, 2017; AGRELI HF, et al., 2016).

À medida que há centralização do cuidado à pessoa e as suas necessidades de saúde, os profissionais passam a direcionar a prática para um horizonte mais amplo, requerendo um trabalho com articulação de ações e integração entre os agentes (AGRELI HF, et al., 2016). Neste contexto, torna-se necessária atenção à saúde mental da pessoa, de forma que a temática se desloque do lugar de "doença/transtorno" e sintomas, para o de saúde/saúde mental como aspecto transversal, ampliando o olhar e o conceito de saúde mental e, convergindo com o modelo de atenção psicossocial (AMARANTE P, 2019; BRASIL, 2013).

De acordo com a lógica psicossocial o propósito a ser atingido é o resgate do convívio social e liberdade de escolha do sujeito, em que todos são atores no processo - profissionais e pessoas/familiares - e o cuidado deve ser pautado na prática interprofissional colaborativa e centrada na pessoa/familiar (AGRELI HF, et al., 2016; AMARANTE P, 2019). Tal modelo representa evolução na forma de cuidar, que marca historicamente o rompimento com o modelo psiquiátrico reducionista e $\mathrm{o}$ anseio por um atendimento que possibilite $\mathrm{e}$ considere a integralidade do sujeito (AMARANTE P, 2019; FIDELIS AC, et al., 2018).

Portanto, percebe-se diversas aproximações e similaridades entre a ACP e o modelo de atenção psicossocial, pois ambos preveem o trabalho colaborativo, voltado às necessidades da pessoa, respaldados em conceitos como autonomia, autocuidado, cidadania, protagonismo e subjetividade (AGRELI HF, et al., 2016; AMARANTE P, 2019).

Tornam-se notórios os avanços nas recomendações e políticas nacionais e internacionais direcionadas à necessidade do olhar ampliado para a saúde mental nos dispositivos de atenção à saúde (ALMEIDA JMCD, 2019; BRASIL, 2013; ESTEVAM AS, et al., 2020).

Entretanto, ainda é pouco compreendida a importância do cuidado em saúde mental no âmbito da APS. Ressalta-se ainda, que como membro da equipe mínima da ESF, o enfermeiro tem importante papel no desempenho de ações interprofissionais e centradas nas necessidades da pessoa/familiar (BRASIL, 2017; SILVA MCM, et al., 2018). Neste contexto, o presente artigo objetiva relatar a experiência de uma discente do Curso de Graduação em enfermagem quanto ao desenvolvimento de intervenções em saúde mental direcionadas a uma adolescente no âmbito da Atenção Primária à Saúde.

\section{RELATO DE EXPERIÊNCIA}

Trata-se de um relato de experiência, que compreende uma pertinente ferramenta de explanação de vivências científicas, hábil a expressar significados intrínsecos (DALTRO MR e FARIA AA, 2019), desenvolvido a partir do estágio curricular do curso de graduação em enfermagem de uma universidade pública em uma ESF de Cuiabá-MT, em julho e agosto de 2019.

Os cuidados direcionaram-se a uma adolescente de quinze anos, que buscou a ESF com queixa de crises convulsivas, com possível causa emocional, para confirmação diagnóstica. Esta residia com a mãe, padrasto e três irmãos, e mantinha pouco contato com o pai biológico. Na ocasião, cursava o $9^{\circ}$ ano do ensino fundamental, mas estava afastada da escola devido à greve dos docentes. Antes disso, frequentava a instituição regularmente.

Após o acolhimento, a adolescente foi convidada a realizar um acompanhamento com a enfermagem, direcionado ao caso índice e estendido a sua família, tendo em vista o respeito à ACP e ao modelo de atenção psicossocial, estabelecidos como referenciais para as ações desempenhadas. 
A experiência abrangeu cinco encontros, entre atendimentos individuais na unidade, exigindo anamnese detalhada, exame físico exame mental, visita domiciliária e, discussão interprofissional com a equipe de Saúde da Família (ESF). Visando conhecer o contexto da usuária, foram utilizadas ferramentas como genograma e ecomapa e durante todo o acompanhamento, foi empregada como estratégia, a comunicação terapêutica.

Resguardando-se à ética, o relato não apresenta qualquer descrição que permita a identificação da pessoa e família, tendo como finalidade descrever a experiência na visão da discente, o percurso metodológico e as principais intervenções realizadas.

O genograma demonstrou agravos à saúde da família em três gerações, excluindo um possível histórico de epilepsia. O ecomapa possibilitou identificar os principais vínculos do núcleo familiar com os parentes, amigos e demais dispositivos da Rede de Atenção à Saúde, para que fosse possível não só identificá-los, como também acioná-los como parceiros a depender da necessidade.

Por meio do ecomapa, foi permitido observar que o irmão da adolescente estabelecia um vínculo forte com a escola de futebol do bairro. Quanto à mãe evidenciou-se um vínculo médio com o Centro de Referência de Assistência Social (CRAS) e vínculo médio da adolescente com a escola, assim como, queixa de relações conflituosas com a instituição. O ecomapa permitiu visualizar também um vínculo fraco da família em relação à eSF e com o pai da adolescente.

Buscou-se realizar uma abordagem inicial individual para a construção do ecomapa, posteriormente agrupada em um ecomapa familiar, possibilitando que dispositivos, nos quais apenas um dos membros da família possuísse vínculo, como o CRAS, pudesse ser estimulado aos demais familiares. A construção dos instrumentos associada às visitas domiciliares permitiu verificar potencialidades dessa família, como a boa interação intrafamiliar e ainda, vulnerabilidades, como vínculo fraco com a ESF.

Considerando que o caso estava em investigação diagnóstica médica e discussão em equipe, a consulta de enfermagem se deu para investigar a queixa principal, complementar o histórico de vida, realizar o acolhimento da adolescente e família, verificar a ocorrência das crises convulsivas e conhecer/identificar os aspectos emocionais envolvidos nessas ocasiões.

Embora a priori, a adolescente negasse qualquer relação das crises convulsivas a fatores psíquicos/emocionais, realizar uma linha cronológica, por meio da conversa com a usuária e sua mãe, permitiu visualizar uma possível relação entre esses eventos (Quadro 1).

Quadro 1 - Caracterização das crises convulsivas referidas, 2019.

\begin{tabular}{|c|c|}
\hline $\begin{array}{c}\text { Ordem } \\
\text { cronológica }\end{array}$ & Fatores emocionais envolvidos \\
\hline $1^{\circ}$ & Ocorreu durante uma briga com a mãe; \\
\hline $2^{\circ}$ & Ocorreu alguns dias após a menarca; \\
\hline $3^{\circ}$ & Ocorreu alguns dias após demissão da mãe; \\
\hline $4^{\circ}$ & Ocorreu dois meses após mudar de cidade, frente a conflitos e dificuldades de \\
adaptação.
\end{tabular}

Fonte: Moraes LG, et al., 2020.

Ainda durante a primeira consulta de enfermagem, a adolescente referiu ideação suicida ativa. Relatou que a ideação iniciou há dois anos, associada à tristeza, ansiedade e irritabilidade. Revelou também episódios de autolesão, sendo o último, seis meses antes da consulta e, desejo de interromper a prática.

Nesta ocasião, foi discutido com a equipe e a adolescente, a possibilidade de iniciar um acompanhamento psiquiátrico e/ou psicoterápico, porém, ela negou-se a realizá-lo, justificando dificuldade em expressar-se e estabelecer diálogo com profissionais desconhecidos. Logo, mantivemos as consultas de enfermagem para acompanhamento em saúde mental, buscando exercer postura acolhedora e estabelecer espaço de escuta qualificada.

Neste contexto, as intervenções foram direcionadas a adolescente e sua família, ao encontro das demandas levantadas, sendo algumas destas observadas a seguir (Quadro 2). 
Quadro 2- Avaliação e Plano de Cuidados, 2019.

\begin{tabular}{|c|c|c|}
\hline $\begin{array}{l}\text { Levantamento de } \\
\text { Problemas }\end{array}$ & Intervenção & Métodos de intervenção \\
\hline \multirow{7}{*}{$\begin{array}{l}\text { Ideação suicida } \\
\text { ativa }\end{array}$} & $\begin{array}{c}\text { Realizada atitude acolhedora, apresentando a unidade, até o momento pouco frequentada pela } \\
\text { adolescente e sua família, como local de produção de saúde e de apoio. Houve o } \\
\text { estabelecimento da escuta ativa, valorizando todas as queixas apresentadas, mantendo o } \\
\text { contato visual e eximindo qualquer dúvida existente. Buscou-se utilizar uma comunicação clara } \\
\text { e sem preconceitos, com estímulo a manutenção da vida. }\end{array}$ & Orientação a autorreflexão \\
\hline & $\begin{array}{c}\text { Realizada estratificação do Risco de Suicídio, com o resultado final de risco elevado. } \\
\text { Encaminhada ao serviço especializado. }\end{array}$ & Escala \\
\hline & $\begin{array}{l}\text { Adolescente convidada a comparecer as consultas para que familiarize a se expressar, } \\
\text { facilitando a adaptação com a psicóloga. Orientada quanto à importância do acompanhamento } \\
\text { psicológico e de serviço especializado para melhoria dos sintomas. }\end{array}$ & Orientação a autorreflexão \\
\hline & $\begin{array}{l}\text { Adolescente orientada a evitar a autolesão, explicado as consequências desses atos e outras } \\
\text { formas de alívio da ansiedade e irritabilidade, tais como banhos quentes, músicas relaxantes, } \\
\text { técnicas de respiração, prática de atividades físicas. }\end{array}$ & Orientação \\
\hline & $\begin{array}{l}\text { Família orientada a implementar precauções contra suicídio, como a retirada de meios letais } \\
\text { (tais como: faca, armas, medicamentos, lâminas) pelos quais a adolescente poderia se ferir, } \\
\text { além de evitar acesso aos meios de suicídio. }\end{array}$ & Orientação \\
\hline & $\begin{array}{c}\text { Realizada, juntamente com a adolescente, a confecção de uma "Lista de Segurança" com o } \\
\text { número de telefone de quatro pessoas de confiança para contatar em casos de planejamento } \\
\text { de suicídio ou quando se sentir sozinha, indicando a eSF e o Centro de Valorização à Vida } \\
\text { (CVV) como lugar/espaço de acolhimento e escuta. }\end{array}$ & Orientação e explanação da rede de apoio \\
\hline & $\begin{array}{l}\text { Realizada discussão com a médica da unidade e encaminhamento para a psicologia em serviço } \\
\text { especializado. }\end{array}$ & $\begin{array}{l}\text { Encaminhamento e proposta de atendimento } \\
\text { interprofissional com a psicologia }\end{array}$ \\
\hline $\begin{array}{l}\text { Convulsões } \\
\text { referidas }\end{array}$ & $\begin{array}{l}\text { Orientada a escrever em um diário seus sentimentos e emoções para confirmar se as crises } \\
\text { convulsivas têm associação com demandas emocionais. }\end{array}$ & Orientação \\
\hline \multirow{3}{*}{ Isolamento social } & Orientada a evitar o isolamento social, por meio do contato com os amigos e família. & Orientação \\
\hline & Instruída a realizar atividades que lhe dão prazer, com pessoas que goste. & Orientação e explanação do Ecomapa \\
\hline & $\begin{array}{c}\text { Família orientada manter vínculo com a adolescente, e informada quanto à importância desse } \\
\text { apoio para a mesma. }\end{array}$ & Orientação \\
\hline
\end{tabular}

Fonte: Moraes LG, et al., 2020.

$$
\text { apoio para a mesma. }
$$


A adolescente participou ativamente dos cuidados, afirmou sentir-se melhor com o acompanhamento, concordou em comparecer as consultas com o profissional de psicologia e referiu reconhecer a ESF como componente de sua rede de apoio. Foi proposto a não repetição de autolesão, se engajar em atividades físicas na escolinha de futebol que o irmão frequenta e escrever um diário, como opções ao desejo de provocar autolesão ou isolar-se.

Embora ao término da atividade curricular, ainda não havia conclusão do diagnóstico médico, foi possível identificar demandas a partir da ACP e o cuidado biopsicossocial, em que as intervenções resultantes da integração entre profissionais, adolescente e sua família possibilitaram melhorias referentes à afetividade, disposição ao autocuidado, humor, e empenho em comunicar/interagir com a acadêmica.

\section{DISCUSSÃO}

A atitude acolhedora permeou todo o cuidado, por meio de uma postura respeitosa, empenhada, valorizando as informações trazidas pelas pessoas envolvidas e, considerando-os como indivíduos singulares, que repercutiu na possibilidade de ofertar um atendimento humanizado, constituir trocas exitosas e, estabelecer um plano de cuidados mais resolutivo (CANDEIAS R, et al., 2019; LOPES et al., 2015).

Este plano esteve pautado na atenção psicossocial e na $A C P$, deslocando a prática de um modelo biomédico para o cuidado do sujeito em sofrimento, suas condições de vida e saúde (AMARANTE P, 2019). A ACP é um dos domínios essenciais para o trabalho interprofissional, por preocupar-se com a subjetividade, participação dos indivíduos, humanização, relações sociais e, agregado ao modelo de atenção psicossocial, tornam-se direcionadores fundamentais para o cuidado na APS (BRASIL, 2017; AGRELI HF, et al., 2016).

O cuidar é exercido neste movimento de reconhecer o outro, e se dá quando a pessoa assistida adquire significado para todos os envolvidos e, à medida que o profissional esteja disponível a participar e entender suas escolhas e sofrimentos, do mesmo modo que a pessoa apresenta disponibilidade para compartilhar sua vida (FIDELIS AC, et al., 2018).

Dessa forma, ao respeitar as decisões da adolescente no âmbito terapêutico, foi possível propiciar o espaço de fazer "com" o outro, em que estar com a adolescente passou a ter um significado de caráter relacional, com compromisso na construção do vínculo, acolhimento e ACP, favorecendo seu acompanhamento (FIDELIS AC, et al., 2018).

Dentre as diferentes estratégias utilizadas, o genograma e o ecomapa possibilitaram a participação da adolescente e sua família, sendo o primeiro uma ilustração da configuração familiar por gerações, com os principais agravos à saúde da parentela e, o segundo, uma representação gráfica das relações estabelecidas com as pessoas e dispositivos de suporte, observando as conexões sociais (SOUZA ÍPD, et al., 2016). Estes foram direcionadores da discussão do caso em equipe, por possibilitarem o conhecimento sistematizado das condições de vida, saúde e vínculos da adolescente e de seus familiares.

No que se refere a ideação suicida explicitada pela adolescente, estas relações (vínculos familiares/sociais e rede) assumem caráter ainda mais importante, interferindo negativamente, aumentando o risco para 0 suicídio, ou positivamente, estabelecendo-se como fator de proteção, por isso, as intervenções devem englobar a família como um todo, estimulando relações saudáveis e seguras (MAGNANI RM e STAUDT ACP, 2018).

A ideação suicida faz parte de um continuum denominado comportamento suicida, que diz respeito a conduta humana de desejar morrer, planejar a morte, ferir-se ou matar-se; permeando conceitos de ideação suicida, tentativa de suicídio e suicídio. A Ideação suicida refere-se a pensar e planejar o suicídio; na tentativa de suicídio, o indivíduo põe em prática seu planejamento, ou seja, objetiva a morte, que acaba não ocorrendo. Para configurar suicídio, o ato contra si deve ser intencional e resultar em morte (MAGNANI RM e STAUDT ACP, 2018).

Importante enfatizar que além de ideias suicidas, foi evidenciado também autolesão por meio de cortes. A autolesão difere do suicídio por ocorrer quando a pessoa não tem intenção de se matar, mas machuca a si próprio para alívio da dor emocional ou para alcançar mudanças desejadas, configura-se um importante fator de risco para o suicídio (ALMEIDA RS, et al., 2018). 
Embora na infância e adolescência o comportamento suicida frequentemente relaciona-se a problemáticas como comportamento impulsivo, descontentamento com o corpo, vínculos interrompidos, disfunção familiar, ocorrência de suicídio e depressão familiar, problemas escolares e transtornos mentais prévios, a compreensão do suicídio envolve distintos fatores inter-relacionados, não devendo ser imputadas causas únicas (SILVA L, 2019).

Portanto, seria reducionista, diante de um comportamento tão complexo, buscar causas ou fatores isolados. Logo, o cuidado à pessoa nessas condições deve ocorrer por meio do acolhimento empático com identificação do estado mental, dos fatores de risco e/ou de proteção, da avaliação de risco para o suicídio e ativação da rede de apoio na tentativa de prevenir um desfecho fatal.

Outro aspecto da vida da adolescente abordado refere-se às crises convulsivas e uma possível relação destas com fatores emocionais. Nesse sentido, as "crises não epilépticas psicogênicas", que geralmente estão associadas a histórico de violência, problemas familiares, baixa condição socioeconômica, luto, tensão, preocupação excessiva, presença de transtornos psíquicos, dentre outras situações, foi vislumbrada como possibilidade (CARTER A, et al., 2018).

Nestas crises, não há identificação de causas orgânicas vinculadas à epilepsia, pois expressam-se por elementos psicoemocionais como originadores da sintomatologia (GOMES LMK e GOMES RF, 2015). Assim como a Somatização, quadro frequente na APS, caracterizado por sintomas físicos, sem a identificação de causas biológicas, pois tem origem no sofrimento mental/emocional vigente (BRASIL, 2013).

A somatização relaciona-se a dificuldade de o indivíduo verbalizar o que está afligindo-o, com repercussões corporais negativas, induzindo a necessidade de investigar o problema prioritário e estabelecer relações de segurança para detectar métodos de enfrentamento e resolução (TORRES ACDAR, et al., 2017).

Portanto, no caso citado, não havia um diagnóstico médico conclusivo e, tal fato, não foi limitante para o cuidado, considerando que a partir do modelo de atenção psicossocial a "doença" é colocada entre parênteses $e$ atende-se à pessoa com suas demandas singulares (AMARANTE $P, 2019$ ). Tais intervenções reforçam a possibilidade de atuação dos profissionais da APS com o olhar não apenas para os sintomas, mas ao seu significado no contexto de vida, de relações familiares e sociais.

Tal olhar que valoriza os aspectos psicossociais dos indivíduos é um dos maiores obstáculos para que os profissionais realizem um atendimento de saúde mental mais acolhedor na APS, ocasionalmente relacionados à falta de preparo, pouca/nenhuma formação em serviço, receio, estigma, recursos materiais limitados e reprodução do modelo biomédico-psiquiátrico (SILVA AFD, et al., 2018).

A perspectiva de cuidado ampliado e pautado na ACP pode fortalecer o cuidado em saúde mental na APS, visto que se destina a responder às necessidades da pessoa, deslocando o cuidado restrito à dimensão patológica para a subjetividade dos sujeitos, integrando ações de promoção, prevenção, recuperação e reabilitação da saúde (AGRELI HF, et al., 2016).

Torna-se imprescindível romper com o modelo biomédico-psiquiátrico e se propor a observar outras esferas da vida da pessoa, para que seja possível prestar uma assistência mais abrangente e integral (SILVA MDFF, et al., 2018).

Superar tal modelo requer reconhecer o indivíduo e sua família como essenciais ao tratamento, esforçarse a manter relações positivas destes com o serviço e, buscar articulações com outros dispositivos da rede, utilizando para isso, ferramentas como genograma e ecomapa, por exemplo (AMARANTE P, 2019). Nesse sentido, esta experiência permitiu-nos visualizar a complexidade desse atendimento e sugerir intervenções junto à adolescente por meio de um plano vinculado à realidade desta, por contar com uma investigação sistematizada e participação dos envolvidos em todo o processo.

Ademais, um acolhimento assertivo acontece por meio do esforço para a criação de vínculos, propiciado por um trabalho respaldado no cuidado centrado na pessoa e mediado pela comunicação terapêutica, que abrange não só a comunicação verbal, mas a postura do trabalhador, escuta de queixas, medos e expectativas; identificação de riscos e responsabilização (BRASIL, 2013; CANDEIAS R, et al., 2019). 
Relatar a experiência como discente de enfermagem quanto ao desenvolvimento de intervenções em saúde mental direcionadas a uma adolescente no âmbito da ESF permitiu uma reflexão acerca da possibilidade de atuação do enfermeiro referente aos cuidados com a saúde mental dos indivíduos na APS, à luz do modelo de atenção psicossocial e ACP, identificados como referenciais necessários neste contexto. Tais propostas são substitutivas ao modelo biomédico-psiquiátrico, por priorizar o vínculo, singularidade, autonomia e novas possibilidades terapêuticas, ampliando o olhar para os sujeitos, seus territórios afetivos e para as ferramentas de cuidado.

\section{REFERÊNCIAS}

1. AGRELI HF, et al. Atenção centrada no paciente na prática interprofissional colaborativa. Interface-Comunicação, Saúde, Educação, 2016; 20: 905-916.

2. ALMEIDA JMCD. Política de saúde mental no Brasil: o que está em jogo nas mudanças em curso. Cadernos de Saúde Pública, 2019; 35(11), 1-6.

3. ALMEIDA RS, et al. A prática da automutilação na adolescência: o olhar da psicologia escolar/educacional. Caderno de Graduação- Ciências Humanas e Sociais, 2018; 4(3): 147-160.

4. AMARANTE P. Saúde Mental e Atenção Psicossocial. 4ed. Rio de Janeiro: Editora Fiocruz, 2019. 123p.

5. BRASIL. Ministério da Saúde. Caderno de Atenção Básica, n. 34: Saúde mental. Brasília (DF): Secretaria de Atenção à Saúde. Departamento de Atenção Básica. Ministério da Saúde, 2013.

6. BRASIL. Portaria no 2.436, de 21 de setembro de 2017. Aprova a Política Nacional de Atenção Básica, estabelecendo a revisão de diretrizes para a organização da Atenção Básica, no âmbito do Sistema Único de Saúde. Diário Oficial da União, 2017.

7. CANDEIAS R, et al. Acolhimento na Estratégia Saúde da Família: concepções e práticas. Revista Norte Mineira de enfermagem, 2019; 8(1): 49-57.

8. CARTER A, et al. Experience of psychogenic nonepileptic seizures in the Canadian league against epilepsy: A survey describing current practices by neurologists and epileptologists. Seizure, 2018; 61: 227-233.

9. DALTRO MR, FARIA AA. Relato de experiência: Uma narrativa científica na pós-modernidade. Estudos e pesquisa em psicologia, 2019; 19(1): 223-237.

10. ESTEVAM AS, et al. A enfermagem em saúde mental pós reforma psiquiátrica. Revista Eletrônica Acervo Saúde, 2020; (45), e2631-e2631.

11. FIDELIS AC. Sentido do cuidado em saúde mental: sobre a rede de atenção psicossocial do sistema único de saúde (SUS). Trabalho, educação e saúde, 2018; 16(2): 561-582.

12. GOMES LMK, GOMES RF. Crises epilépticas $x$ crises não epilépticas psicogênicas: diagnóstico diferencial. Revista Caderno Pedagógico, 2015; 12(1), 196-207.

13. LOPES AS, et al. O acolhimento na Atenção Básica em saúde: relações de reciprocidade entre trabalhadores e usuários. Saúde debate, 2015; 39(104), 114-123.

14. MAGNANI RM, STAUDT ACP. Estilos parentais e suicídio na adolescência: uma reflexão acerca dos fatores de proteção. Pensando famílias, 2018; 22(1): 75-86.

15. SILVA AFD, et al. Acolhimento à pessoa em sofrimento mental na atenção básica. Revista de enfermagem UFPE online, 2018; 12(9): 2459-2469.

16. SILVA L. Suicídio entre crianças e adolescentes: um alerta para o cumprimento do imperativo global. Acta Paulista de Enfermagem, 2019; 32(3): 3-6.

17. SILVA, MCM, et al. Saúde mental em uma unidade de atenção primária à saúde: relato de experiência. Ciência \& Saúde, 2018; 11(1), 65-69.

18. SILVA MDFF, et al. Integralidade na atenção primária à saúde. Revista Família, Ciclos de Vida e Saúde no Contexto Social, 2018; 6 (Supl. 1): 394-400.

19. SOUZA ÍPD, et al. Genograma e ecomapa como ferramentas para compreensão do cuidado familiar no adoecimento crônico de jovem. Texto \& contexto enfermagem, 2016; 25(4): 1-10.

20. TORRES ACDAR, et al. A somatização de um sofrimento: assistência de enfermagem nos fenômenos psicossomáticos em pacientes com câncer de mama. Revista Interdisciplinar Pensamento Científico, 2017; 3(2): 356277. 